\title{
Synthesis of TiN Reinforced Aluminium Metal Matrix Composites Through Microwave Sintering
}

\author{
K. Venkateswarlu, Suman Saurabh, V. Rajinikanth, Ranjan Kumar Sahu, and Ajoy Kumar Ray
}

(Submitted December 12, 2008; in revised form March 12, 2009)

\begin{abstract}
Al-TiN (10, 20, 30 wt.\%) composites were fabricated by using microwave radiation. Al and TiN powders were selected as starting materials, mixed in a ball mill for $\sim 10$ min and sintered for various times. Results indicate that an optimum microwave sintering time of $2 \mathrm{~min}$ was essential and responsible for the improved densification and mechanical properties. The presence of TiN particles at grain boundaries plays a significant role in improving the densification and hardness values. Dry sliding wear results show the improved wear resistance of the composite (Al-TiN) due to the presence of TiN particles and the wear results are superior to the $\mathrm{Al}-\mathrm{TiN}$ samples made by hot pressing technique.
\end{abstract}

Keywords mechanical properties, metal matrix composite, microstructure, microwave sintering, powder metallurgy

\section{Introduction}

Composite materials are well known for their tailored properties that are achieved by combining two or more materials to obtain the required spectrum of properties. The most distinctive feature of composites is that, while the individual constituents retain their properties, they synergize to give properties which may not be found in any one of them alone. Recently, extensive research study has been carried out, and it was shown that there is tremendous promise of ceramicreinforced metal-matrix composites (MMCs) (Ref 1-3). Various processing techniques have been developed to engineer composites for diverse fields of applications (Ref 1,2) such as aerospace, defense, automobile, and sports sectors. In MMCs, the addition of a small amount of second-phase materials with high shear strength imparts unique properties to the base materials (Ref 4-6). The strength of MMCs is strongly influenced by the shape, orientation, and volume fraction of the second phase. Thus, the different possibilities of topological combinations of the different materials in space have led to the development of different types of composites such as laminated, continuous fiber, reinforced, interpenetrating, discontinuously reinforced etc.

Aluminum-based MMCs have received much attention due to their light weight, high strength-to-weight ratio, ease in melting and casting, and abundantly available raw materials. Most of the research on the Al-based composites is to improve their mechanical properties by the incorporation of hard ceramic oxides/carbides/nitrides into the metal matrix. Among various

K. Venkateswarlu, National Aerospace Laboratories, Bangalore 560017, India; V. Rajinikanth, Ranjan Kumar Sahu, and Ajoy Kumar Ray, National Metallurgical Laboratory, Jamshedpur 831007, India; and Suman Saurabh, National Institute of Technology, Tiruchirapalli, Tamilnadu 620015, India. Contact e-mails: karodi2002@ yahoo.co.in and rajni@nmlindia.org.

processing techniques, the powder metallurgy route has been extensively studied. The process includes cold-isostatic pressing (CIP) followed by sintering (Ref 7), hot-pressing (Ref 8), and hot-isostatic-pressing (HIP) (Ref 9) techniques. Another route of synthesis is via a liquid metallurgy route where the reinforcing phases are either infiltrated with (Ref 10-12) or injected into the liquid metal. Mixing, in situ growth, and spray-forming techniques are also used to prepare the MMCs. The spray forming of composites involves the forming of composites by spraying liquid metal and reinforcing particulates simultaneously (Ref 13-15). Among various ceramic additions to Al, $\mathrm{SiC}$ has been given much greater attention by many researchers but the Al-SiC still has some limitations such as the formation of $\mathrm{Al}_{4} \mathrm{C}_{3}$ during high temperatures which impairs the mechanical properties of the Al MMCs (Ref 1). Thus, efforts are made to overcome the problems by incorporating other reinforcing materials such as TiN (Ref 16) and also through innovative synthesizing techniques like microwave sintering (Ref 17-20).

Microwave sintering ( $\operatorname{Ref} 21,22$ ) has been recognized as a time-saving and cost-effective process as compared to the conventional powder metallurgy processes for synthesizing composite materials. In the normal mode of resistance heating, the direction of heat flow is from outside to inside of the powder compact. This leads to inferior microstructural properties of the core. In contrast, for microwaves, the direction of heating or heat flow is from the inside of the powdered compact to the outside. However, this does not yield very good microstructural properties of the surface. Thus, both these methods are not perfect to yield a good overall property of the sample. A combined action of microwaves and microwave-coupled external heating has therefore been proposed to overcome the drawbacks of both these sintering methods, to realize rapid sintering from both outside and inside of the powdered compact. A microwave susceptor material such as $\mathrm{SiC}$ is, therefore, used to assist sintering from outside (Ref 16,22 ).

This article mainly deals with three aspects of the microwave synthesis of composites: (1) incorporating TiN in Al matrix in place of $\mathrm{SiC}$, (2) overcoming the problems from melting, sprayforming and powder metallurgy routes by opting for the microwave-sintering technique, and (3) comparing the improvement in mechanical properties with our earlier studies (Ref 23). 


\section{Experimental Details}

Air-atomized aluminum powder of $99.7 \%$ purity, globular copper powder of $99.5 \%$ purity, and TiN powders were selected as the starting materials. Hereafter, $\%$ is always reported as wt. $\%$. The TiN powder was prepared by nitriding the Ti sponge ( $>98 \%$ pure) at $1823 \mathrm{~K}$ for $45 \mathrm{~min}$. After nitridation, the lumpy mass was manually ground to yield powder by using an agate mortar. The powder was again nitrided and reground to ensure the complete nitridation of $\mathrm{Ti}$ and the formation of a single-phase TiN powder. The process of nitridation and grinding was repeated three times (Ref 23). Al powder (99.7\% purity) was prepared by air-atomization technique in our laboratory using a graphite nozzle of 3-mm diameter and gas pressure of $50 \mathrm{psi}$ and sieved to $\sim 50 \mu \mathrm{m}$. Copper powder (99.5\% purity) was used to improve the wettability of $\mathrm{Al}$ and TiN (Ref 23). The required amounts of $\mathrm{Al}, \mathrm{TiN}$, and $\mathrm{Cu}$ powders were taken in three separate batches with the compositions given in Table 1. The powder mixture was thoroughly mixed using agate motor, and cylindrical pellets of diameter $22 \mathrm{~mm}$ were prepared by applying a pressure of $200 \mathrm{MPa}$. Subsequently, the pellets were transferred to a specially designed double-walled reactor consisting of two chambers, inner and outer and placed at the center of a microwave oven (Fig. 1). The samples were kept in the inner chamber and enclosed by the outer chamber filled with $\mathrm{SiC}$ susceptor since it is a good microwave absorber ( $\varepsilon 10.0)$. Since the $\mathrm{SiC}$ susceptor is larger as compared to the sample size, and the microstructures after sintering at edge, center, and another edge are uniform, it is believed that sintering occurred uniformly. The reaction chamber also had gas purging facility. Argon gas of LR1 grade supplied by BOC, Jamshedpur, India

Table 1 Various compositions of Al-TiN MMCs with $4 \% \mathrm{Cu}$

\begin{tabular}{llc}
\hline & \multicolumn{2}{c}{ Composition, wt.\% } \\
\cline { 2 - 3 } Sample & Al & TiN \\
\hline A & 86 & 10 \\
B & 76 & 20 \\
C & 66 & 30 \\
\hline
\end{tabular}

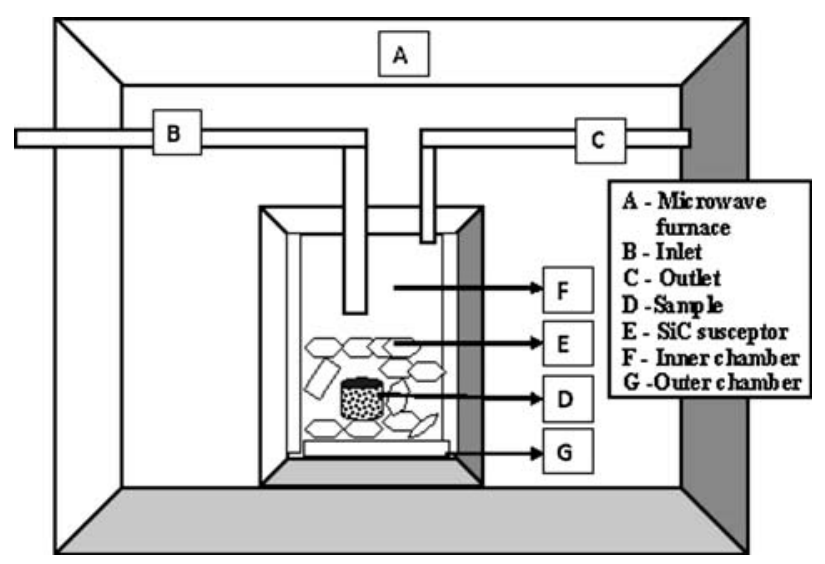

Fig. 1 Schematic diagram of double-walled setup in the microwave oven was passed through the inlet tube. The whole reactor set up was maintained in an argon atmosphere and sintering was carried out several times. The samples were sintered in a continuous supply of argon for $1,2,3$, and $4 \mathrm{~min}$, respectively, and allowed to cool for $10 \mathrm{~min}$ in the microwave oven.

The sintered samples were taken out of the reactor after closing the gas supply. It was observed that the shrinkage of pellets increases with increase in sintering time resulted in higher densification. The sintered samples were polished and prepared for hardness determination and metallographic examination. Both the bulk hardness of Al-TiN composite and micro hardness of individual phases including the interface was determined using Vickers hardness tester and Leitz micro hardness tester, respectively. The samples were etched with standard Keller's reagent and examined under both optical microscope and SEM (JEOL 840A). The chemical compositions of the individual phases were confirmed using energy dispersive $x$-ray spectroscopy (EDS). Dry sliding wear test was carried out on a pin-on-disc wear test machine (TR $20 \mathrm{LE}$, DUCOM, Bangalore, India) with a sample of 8-mm diameter and 40-mm length. The sintered pellet has a height of $5 \mathrm{~mm}$ which has been raised to $40 \mathrm{~mm}$ by adhering the samples to a steel sample of 8-mm diameter and 35- $\mathrm{mm}$ height. A thermocouple was inserted at a height of $2.5 \mathrm{~mm}$ from the bottom of sample for recording the variation in temperature during sliding test. The specimens were allowed to slide against a rotating EN 32 steel disc (counter face) of hardness $65 \mathrm{Rc}$, where coefficient of friction, temperature, and wear were monitored as a function of sliding distance up to $3200 \mathrm{~m}$ at 1 and $2 \mathrm{~kg}$ loads.

\section{Results and Discussion}

Figure 2 shows the XRD pattern for the experimental alloys A, B, and C. Samples A, B, and C denote Al-10\%TiN, $\mathrm{Al}-20 \% \mathrm{TiN}$, and $\mathrm{Al}-30 \% \mathrm{TiN}$, respectively. All the samples showed the presence of TiN peaks along with $\mathrm{Al}$ peaks. It is

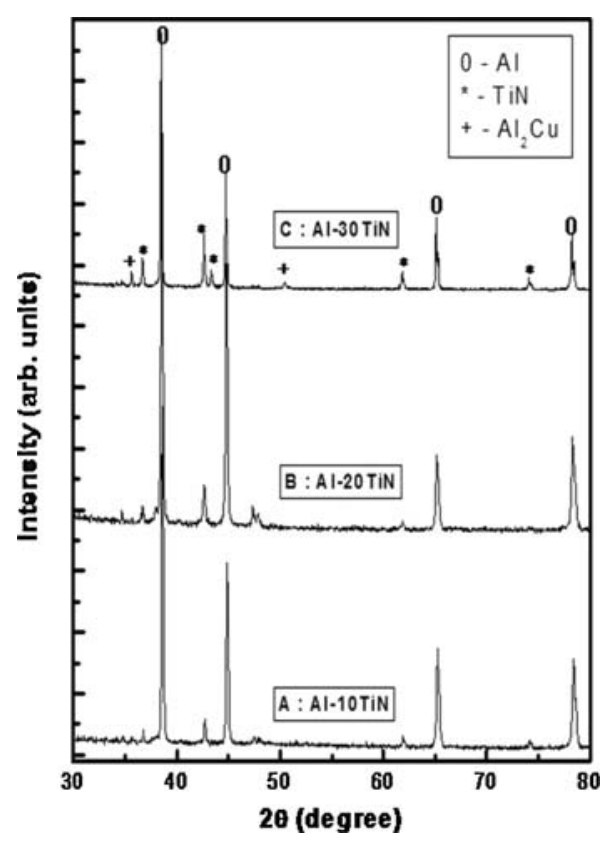

Fig. 2 The XRD pattern for samples A, B, and C 

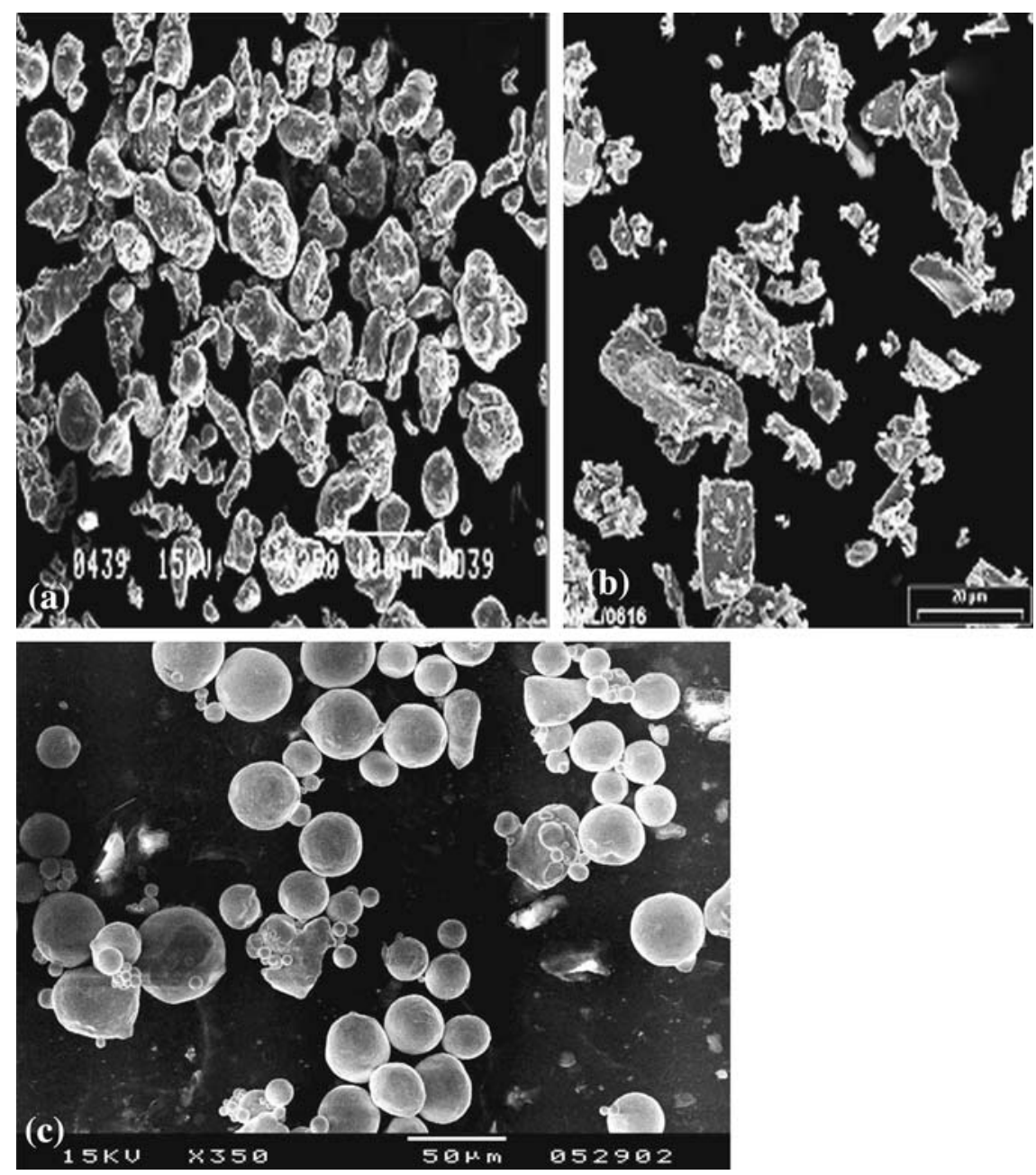

Fig. 3 (a) SEM image of atomized Al powder, (b) SEM image of TiN powder, and (c) SEM image of Copper powder

clearly observed that the intensity of TiN peaks has increased with increase in TiN content from $10 \%$ to $30 \%$. The formation of $\mathrm{Al}_{2} \mathrm{Cu}$ is also observed along with the TiN and $\mathrm{Al}$ peaks in the XRD pattern. There are some peaks for sample B (Al-20TiN) at $2 \theta$ values between $46^{\circ}$ and $48^{\circ}$ and are unidentified. Figure 3(a), (b), and (c) shows the SEM images of Al, TiN, and copper powders, respectively. The average particles size of copper powder is $\sim 25 \mu \mathrm{m}$ and most of the particles are globular in shape. The size distribution of Al particles mostly lies in the range of $40-45 \mu \mathrm{m}$ with some fine particles in the range of 5-8 $\mu \mathrm{m}$. As the TiN powder was prepared by nitriding Ti sponge, it showed porous structure as observed from Fig. 3(b). It is known that such porous structure is more favorable for sintering (Ref 24) and also expected to have better bonding with Al. The particle size distribution of TiN powder exhibited that most $(\sim 75 \%)$ of the particles are in the range of $4-6 \mu \mathrm{m}$. Some TiN particles are agglomerated due to their fineness and are in the range of 1-2 $\mu \mathrm{m}$. Similarly, the average particle size for copper and $\mathrm{Al}$ is in the range of $35-40 \mu \mathrm{m}$.

TiN is a highly stable covalent compound, and hence its wettability with metals and ceramics is very poor. Copper, which is known to increase the wettability of TiN (Ref 23), was added to the Al-TiN mixture. Figure 4(a) and (b) shows the SEM images of samples A and C, respectively. Most of the TiN particles observed are in the range of $4-6 \mu \mathrm{m}$ and marked as TiN
(Fig. 4a). A good interfacial metallurgical bonding is observed between a large TiN particle and $\mathrm{Al}$ matrix taken at higher magnification (Fig. 4c). A close observation also revealed the presence of interfacial phase formation having the composition of $\mathrm{Al}_{2} \mathrm{Cu}$ in the $\mathrm{Al}$ matrix which is responsible for improved wettability. The presence of $\mathrm{TiN}$ and $\mathrm{Al}_{2} \mathrm{Cu}$ phases is confirmed by EDS. There results suggest that some percentage of $\mathrm{Cu}$ is reacting with $\mathrm{Al}$ to form $\mathrm{Al}_{2} \mathrm{Cu}$ during sintering, which is consistent with the results obtained from the XRD studies. No attempt is made to determine the $\mathrm{Al}_{2} \mathrm{Cu}$ content, and therefore, it is difficult to predict how much $\mathrm{Cu}$ reacted with $\mathrm{Al}$ to form $\mathrm{Al}_{2} \mathrm{Cu}$. Moreover, the effective volume fraction of copper that is not reacted with any other elements is less than $\sim 1 \%$ and not detected in XRD studies. The XRD results obtained for the Al-TiN samples are similar to the samples prepared by powder metallurgy route that are observed in our earlier studies (Ref 23).

The mechanical properties (hardness values) of microwavesintered samples are much superior to the earlier reported values (Table 2). It is inferred from the table that the bulk hardness of the samples $\mathrm{A}, \mathrm{B}$, and $\mathrm{C}$ are 58, 73, and $90 \mathrm{Hv}$, respectively. This suggests that microwave sintering is responsible for the enhancement of bulk hardness. Furthermore, microwave sintering is a cost-effective and less time-consuming process. A visual examination followed by hardness testing was carried out for samples sintered for 1, 2, 3, and $4 \mathrm{~min}$. The 

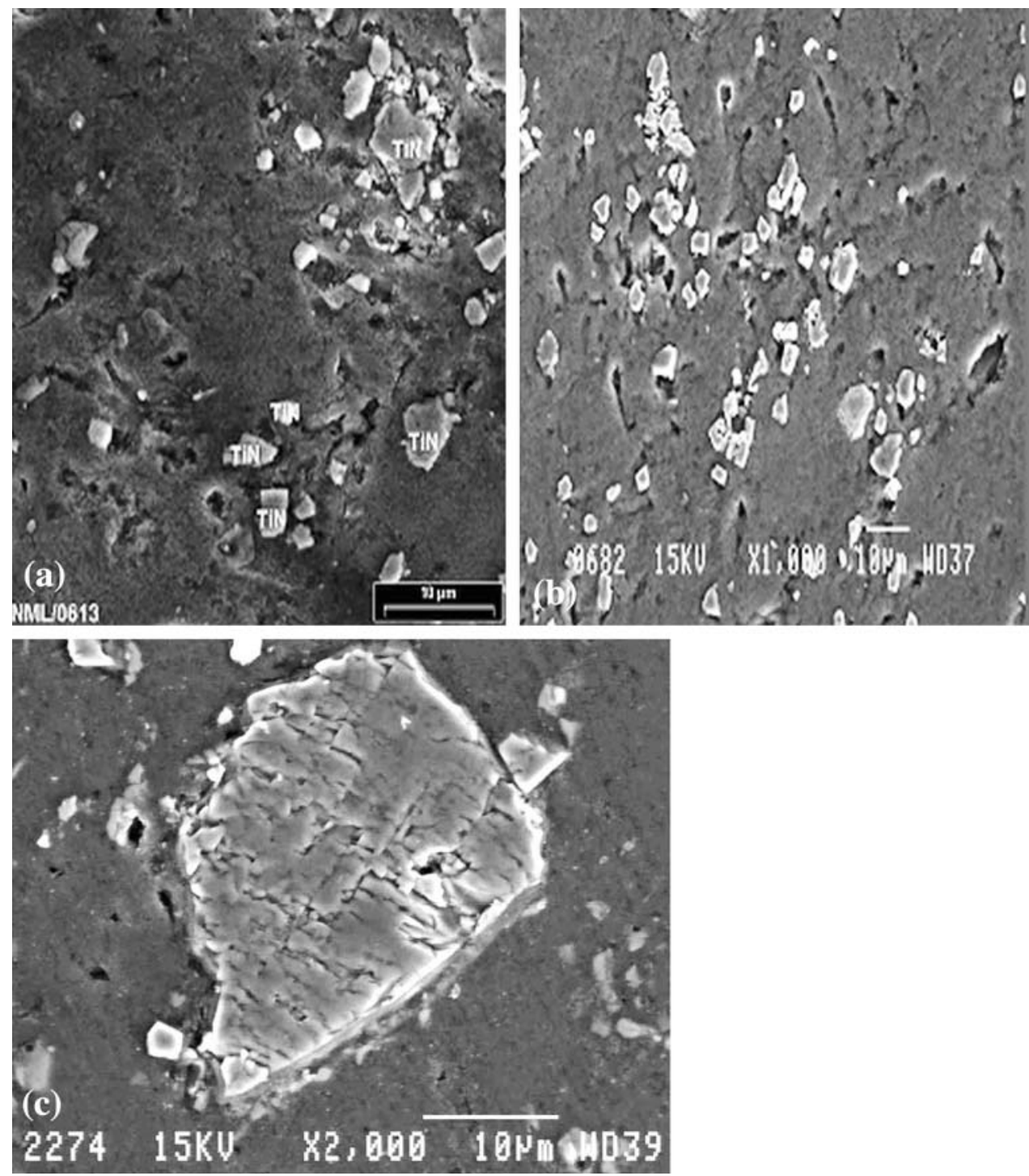

Fig. 4 (a) SEM images of sample A, (b) SEM images of sample C, and (c) shows better bonding of TiN with Al matrix for sample B

Table 2 Hardness values of samples A-C

\begin{tabular}{lccl}
\hline & \multicolumn{2}{c}{ Bulk hardness, Hv } & \\
\cline { 2 - 3 } Sample & $\begin{array}{c}\text { Sintered } \\
\text { (Ref 23) }\end{array}$ & $\begin{array}{c}\text { Microwave } \\
\text { sintered }\end{array}$ & $\begin{array}{c}\text { Microhardness, } \\
\text { Hv }\end{array}$ \\
\hline Al-10TiN-4Cu & 50 & 58 & Matrix $\sim 105$ \\
Al-20TiN-4Cu & $\ldots$ & 73 & Interface $\sim 180$ \\
Al-30TiN-4Cu & 54 & 90 & On Particle $\sim 2500$ \\
\hline
\end{tabular}

samples microwave processed for $1 \mathrm{~min}$ did not sinter whereas the samples processed for 2, 3, and 4 min showed excellent sintering behavior and good hardness. However, a sintering time of more than 2 min ( 3 and $4 \mathrm{~min}$ ) caused sweating of $\mathrm{Al}$ from the pellets. Since the trend is similar for all Al-TiN (10, 20,30 wt. $\%$ ) samples, we have reported the hardness values only for 2-min-sintered samples. These results further suggest that a reaction time of $2 \mathrm{~min}$ is sufficient for good sintering, and that the higher holding time is responsible for probably exceeding the temperature of $\sim 700{ }^{\circ} \mathrm{C}$ due to which $\mathrm{Al}$ melts out from the pellet. The increased hardness could be attributed to increase in the amount of hard phases (TiN) in the Al matrix. Furthermore, it is observed that Al matrix exhibited higher micro hardness values compared to pure Al sample. This may be due to the distribution of fine TiN particles in the Al matrix. In addition to this, a higher micro hardness value $(\sim 180 \mathrm{Hv})$ was observed at the interface of a large TiN particle as compared to the Al matrix. The possible reason for the higher hardness value could be that the applied load was shared between $\mathrm{Al}$ matrix and $\mathrm{TiN}$ particle. No separate micro hardness measurement was made for $\mathrm{Al}_{2} \mathrm{Cu}$ compound as their concentration is very low. The micro hardness value for TiN particles was $\sim 2500 \mathrm{Hv}$ and is close to the reported value (Ref 25).

Figure 5 shows the wear performance for a continuous sliding distance for samples A-C at two different applied loads, 1 and $2 \mathrm{~kg}$. The wear loss is plotted against the sliding distance. It is clear from Fig. 6 that samples at $2-\mathrm{kg}$ load showed higher wear loss as compared to samples at $1-\mathrm{kg}$ load. Sample C exhibited higher wear resistance as compared to samples A and B. This is expected as higher content of TiN and good 


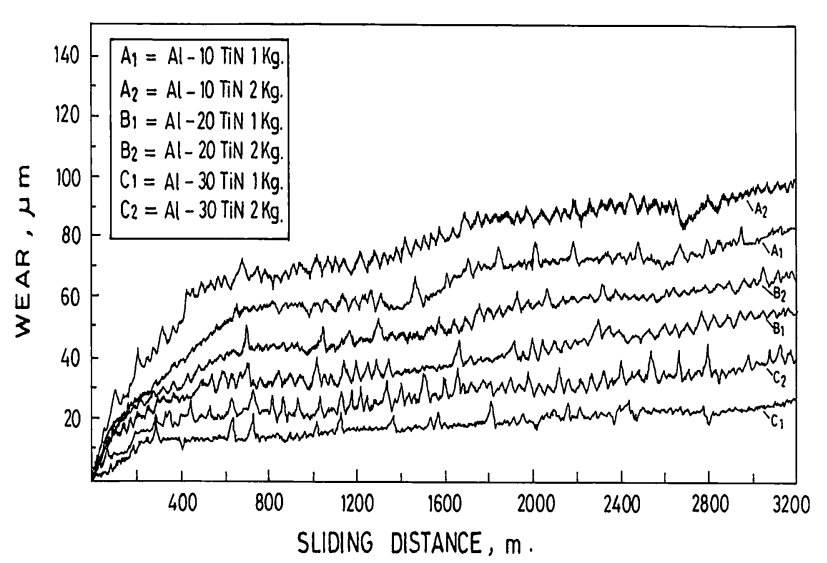

Fig. 5 Sliding Wear behavior of Al-TiN composites

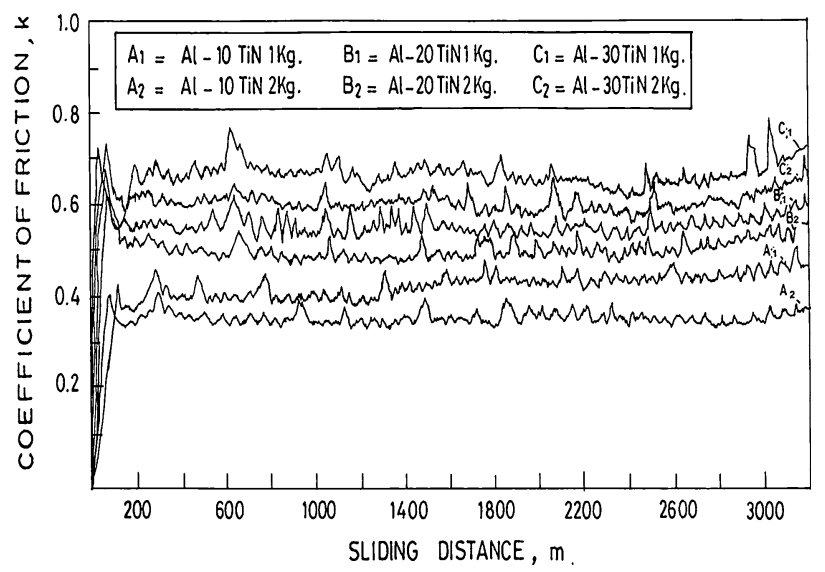

Fig. 6 Coefficient of friction variation with sliding distance

metallurgical bonding with Al matrix resulted in better wear resistance. Figure 6 shows the values of coefficient of friction for a continuous sliding distance of samples A, B, and C. It is observed that initially the coefficient of friction between the sample and steel disc increases steeply up to a maximum value and remains stable until the end of sliding distance of $3200 \mathrm{~m}$. In some cases, a marginal increase in the friction values is also observed. The coefficients of friction $(k)$ for the samples A1 and A2 are ranging from 0.40 to 0.30 , which and are comparatively lower than the samples $\mathrm{C} 1$ and $\mathrm{C} 2$ (0.70 to 0.60$)$. The samples at higher loads showed low coefficient of friction values and is in agreement with the observations made by Grzesik et al. (Ref 26). At higher loads, lower coefficient values are observed for TiAlN coated samples. The temperature variation during sliding wear test is also observed and shown in Fig. 7. The results suggest that initially there is a sudden rise in the temperature up to a sliding distance of $200 \mathrm{~m}$ due to adhesion mechanism between the sample and the disc and beyond that there is a marginal increase in the temperature.

\section{Conclusions}

1. Al-TiN MMCs containing 10, 20, and $30 \mathrm{wt} . \%$ TiN have been successfully synthesized by using microwave

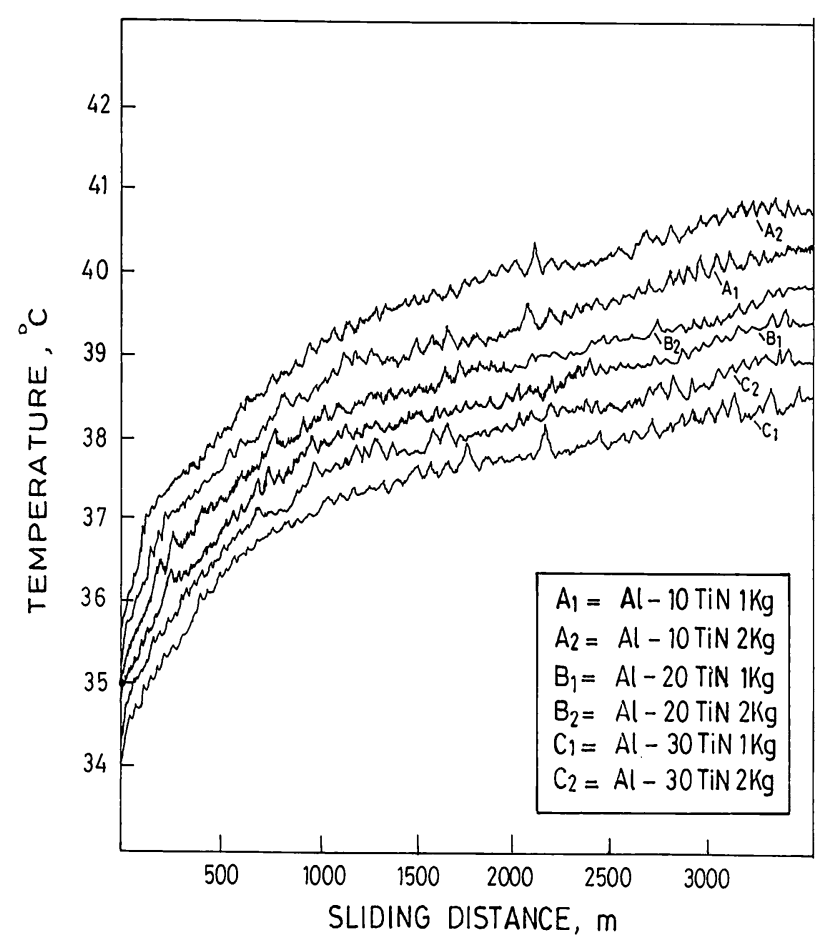

Fig. 7 Variation of temperature with sliding distance

radiation technique. The process of microwave sintering has been considered as the most convenient and economical as compared to other conventional sintering processes.

2. The presence of $\mathrm{SiC}$ susceptor during microwave sintering is responsible for an efficient way of supply of heat to the samples in the microwave oven.

3 . Our experimental results suggest that the microwave sintering of 2 min is sufficient and further increase in sintering time led to melting out of Al from Al-TiN pellet, suggesting that the larger reaction timings are responsible for increase in the reaction time and probably when the temperature reaches more than $\sim 700{ }^{\circ} \mathrm{C}$. The other conventional sintering processes, in general, take few hours for proper sintering.

4. Increasing TiN from 10 to $30 \mathrm{wt} . \%$ showed superior hardness and wear resistance properties as compared to Al-TiN composites prepared by hot pressing.

\section{Acknowledgments}

The authors wish to express their sincere thanks to Prof. S.P. Mehrotra, Director National Metallurgical Laboratory (NML) for his kind permission to publish this article. We sincerely thank Mr. M.K. Gunjan and Mr. B. Mahato for SEM and XRD studies, respectively. We also thank Mr. K. Seetharaman and Mr. Y.P.K. Rao, technical officers, NML, Jamshedpur for fabricating the quartz reaction chamber used in the microwave oven and providing engineering drawing support, respectively. We sincerely thank Prof T.G. Langdon, University of Southern California, USA for his invaluable help in correcting this manuscript. 


\section{References}

1. D.J. Lloyd, Particle Reinforced Aluminum and Magnesium Matrix Composites, Int. Mater. Rev., 1994, 39, p 1-23

2. P.K. Rohatgi, R. Asthana, and S. Das, Solidification, Structure and Properties of Cast Metal-Ceramic Particle Composites, Int. Mater. Rev., 1986, 31, p 115-139

3. I.A. Ibrahim, F.A. Mohammed, and E.J. Lavernia, Particle Reinforced Metal Matrix Composites, J. Mater. Sci., 1991, 26, p 1137-1156

4. A. Kelly, Composites in Context, Compos. Sci. Technol., 1985, 23, p 171-199

5. K. Akio, O. Atsushi, K. Toshori, and T. Horoyuki, Fabrication Process of Metal Matrix Composite with Nano-size SiC Particle Produced by Vortex Method, J. Jpn. Instit. Light Met., 1999, 49, p 149-159

6. R.F. Shyua, F.T. Wenga, and C.T. Hob, In situ Reacted Titanium Nitride-reinforced Aluminum Alloy Composite, J. Mater. Proc. Tech., 2002, 122, p 301-304

7. I.B. Jeong, K.H. Oh, H.I. Lee, D.N. Lee, and Y.K. Baeck, On the Mechanical Behavior of SiC Whisker Reinforced Al Composites by Powder Metallurgy Process, J. De Phys., 1988, 49, p 623-629

8. S. Kohara, Fabrication of SiCp-Al Composite Materials, Mater. Manuf. Process, 1990, 5(1), p 51-62

9. A. Zulfia, H.V. Atkinson, H. Jones, and S. King, Effect of Hot Isostatic Pressing on Cast A357 Aluminum Alloy With and Without SiC Particle Reinforcement, J. Mater. Sci., 1999, 34, p 4305-4310

10. Y. Gao, J. Jia, R.E. Loehman, and K.G. Ewsuk, Transmission Electron Microscopy Study of $\mathrm{Al} / \mathrm{Al}_{2} \mathrm{O}_{3}$ Composites Fabricated by Reactive Metal Infiltration, J. Mater. Res., 1995, 10(5), p 1216-1225

11. B.S. Murty, S.K. Thakur, and B.K. Dhindaw, On the Infiltration Behavior of $\mathrm{Al}, \mathrm{Al}-\mathrm{Li}$ and $\mathrm{Mg}$ Melts through $\mathrm{SiCp}$ Bed, Metall. Mater. Trans. A, 2000, 31A, p 319-325

12. J.Q. Jiang, A.B. Ma, H.N. Liu, and R.S. Tan, Fabrication of Alumina Short Fibre Reinforced Aluminum Alloy Via Centrifugal Force Infiltration, Mater. Sci. Tech., 1994, 10, p 783-788

13. Y. Wu and E.J. Lavernia, Spray Atomization and Co Deposited 6061 Al/SiCp Composites, Metals, 1991, 43, p 16-23

14. W.Y. Li, G. Zhang, H.L. Liao, and C. Coddet, Characterizations of Cold Sprayed TiN Particle Reinforced A12319 Composite Coating, J. Mater. Process. Tech., 2008, 202, p 508-513
15. R. Morgan, P. Fox, J. Pattison, C. Sutcliffe, and W.O. Neill, Analysis of Cold Gas Dynamically Sprayed Aluminum Deposits, Mater. Lett., 2004, 58, p 1317-1320

16. S.K. Thakur, T.S. Kong, and M. Gupta, Microwave Synthesis and Characterization of Metastable (Al/Ti) and Hybrid $(\mathrm{Al} / \mathrm{Ti}+\mathrm{SiC})$ Composites, J. Mater. Sci. Eng. A, 2007, 452, p 61-69

17. R.K. Sahu, A.K. Ray, A.J. Kailath, and L.C. Pathak, Microwaveassisted Combustion Synthesis of Ni Powder Using Urea, J. Mater. Res., 2006, 21, p 1664-1673

18. S.S. Manoharan, S.J. Swathi Prasanna, M.L. Rao, and R.K. Sahu, Microwave-Assisted Synthesis of Fine Particle Oxides Employing Wet Redox Mixtures, J. Am. Ceram. Soc., 2002, 85, p 2469-2471

19. E. Breval, J.P. Cheng, D.K. Agrawal, P. Gigl, M. Dennis, R. Roy, and A.J. Papworth, Comparison Between Microwave and Conventional Sintering of WC/Co Composites, Mater. Sci. Eng. A, 2005, 391, p 285-295

20. P. Yadoji, R. Peelamedu, D. Agrawal, and R. Roy, Microwave Sintering of Ni-Zn Ferrites: Comparison with Conventional Sintering, Mater. Sci. Eng. B, 2003, 98, p 269-278

21. C.-L. Huang and W.-R. Yang, Effect of $\mathrm{CuO}$ Addition to $\mathrm{Nd}$ $\left(\mathrm{Zn}_{1 / 2} \mathrm{Ti}_{1 / 2}\right) \mathrm{O}_{3}$ Ceramics on Sintering Behavior and Microwave Dielectric Properties, Mater. Lett., 2009, 63, p 103-105

22. C. Leonelli, P. Veronesi, L. Denti, A. Gatto, and L. Iuliano, Microwave Assisted Sintering of Green Metal Parts, J. Mater. Process. Tech., 2008, 205, p 489-496

23. A.K. Ray, K. Venkateswarlu, S.K. Chaudhury, S.K. Das, B. Ravi Kumar, and L.C. Pathak, Fabrication of TiN Reinforced Aluminum Metal Matrix Composites Through a Powder Metallurgical Route, Mater. Sci. Eng., 2002, A338, p 160-165

24. A. Herzog, U.F. Vogt, S. Siegmann, and O. Beffort, Aluminium Metal Matrix Composites Based on Biomorphic Silicon Carbide, Adv. Eng. Mater., 2006, 8, p 980-983

25. W.L.E. Wong, S. Karthik, and M. Gupta, Development of High Performance $\mathrm{Mg}-\mathrm{Al}_{2} \mathrm{O}_{3}$ Composites Containing $\mathrm{Al}_{2} \mathrm{O}_{3}$ in Submicron Length Scale Using Microwave Assisted Rapid Sintering, Mater. Sci. Tech., 2005, 31, p 1063-1070

26. W. Grzesik, Z. Zalisz, S. Krol, and P. Nieslony, Investigations on Friction and Wear Mechanisms of the PVD-TiAlN Coated Carbide in Dry Sliding Against Steels and Cast Iron, Wear, 2006, 261, p 11911200 